\title{
OPTICAL AND STRUCTURAL PROPERTIES OF AIGaN/GaN QUANTUM WELLS GROWN BY MOLECULAR BEAM EPITAXY
}

\author{
Nicolas Grandjean*, Jean Massies*, Mathieu Leroux*, Marguerite Laügt*, Pierre Lefebvre**, \\ Bernard Gil**, Jacques Allègre**, Pierre Bigenwald *** \\ * Centre de Recherche sur l'Hétéro-Epitaxie et ses Applications - CNRS \\ Rue B. Grégory, Sophia Antipolis, F-06560 Valbonne, France. \\ ** Groupe d'Etude des Semiconducteurs - CNRS - Université Montpellier II. \\ Case Courrier 074. 34095 Montpellier Cedex 5, France. \\ *** LPM - Université d'Avignon - 33, rue Pasteur. 84000 Avignon, France.
}

Cite this article as: MRS Internet J. Nitride Semicond. Res. 4S1, G11.7 (1999)

\begin{abstract}
AlGaN/GaN quantum well (QWs) were grown on (0001) sapphire substrates by molecular beam epitaxy (MBE) using ammonia as nitrogen precursor. The Al composition in the barriers was varied between 8 and $27 \%$ and the well thickness from 4 to 17 monolayers (MLs, $1 \mathrm{ML}=$

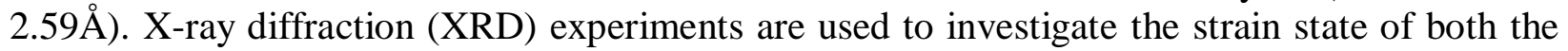
well and the barriers. The QW transition energy are measured by low temperature photoluminescence (PL). A large quantum confined Stark effect is observed leading to QW luminescence much lower than the emission line of the GaN buffer layer for well width above a certain critical thickness. The built-in electric field responsible for such a phenomenon is deduced from fit of the PL data. Its magnitude is of several hundred $\mathrm{kV} / \mathrm{cm}$ and increases linearly with the Al composition.
\end{abstract}

\section{INTRODUCTION}

The III-V nitride compounds have demonstrated their peculiar aptitude for producing light from the green to UV spectral range [1]. As for the case of other optoelectronic devices based on arsenide, phosphide or antimonide compounds, quantum wells (QWs) are currently used in the active region of nitride based light emitting diodes and laser diodes [1]. However, there is still only a few reports on the physical properties of nitride QWs compared to what is known about the prototypical system AlGaAs/GaAs. This is especially the case when considering the $\mathrm{AlGaN} / \mathrm{GaN}$ QWs [2-8] though they appear very promising for extending the applications of nitrides to the far UV spectral region owing to the large direct band gap of AlN $(6.2 \mathrm{eV} / 200 \mathrm{~nm})$. It has been already shown that a large internal electric field of several hundred $\mathrm{kV} / \mathrm{cm}$ takes place in the wurtzite $\mathrm{AlGaN} / \mathrm{GaN}$ QWs [2-8]. Both piezoelectric and spontaneous polarization must be considered to account for the polarization field inside the quantum structure. Actually, spontaneous polarization has been theoretically considered because of the expected huge influence on the properties of $\mathrm{AlGaN} / \mathrm{GaN}$ heterostructures $[9,10]$.

In this paper, we report on $\mathrm{AlGaN} / \mathrm{GaN}$ QWs grown by MBE on c-plane sapphire substrates. Their structural properties have been checked by XRD and their optical properties by low temperature PL. The Al composition of the AlGaN barriers was ranged between 8 and $27 \%$ and 
the well thickness increases from 4 to 17 MLs. The internal electric field was deduced by fitting the PL data using a self-consistent envelope function calculation, including excitonic effects.

\section{EXPERIMENTS}

The growth of GaN and AlGaN layers are carried out in a Riber $32 \mathrm{P}$ MBE system. Both reflection high-energy electron diffraction (RHEED) and laser reflectivity facilities are available in situ. The substrate is c-plane sapphire back-coated with Mo for heating. $\mathrm{NH}_{3}$ is used as nitrogen precursor. $\mathrm{Ga}$ and $\mathrm{Al}$ elements are evaporated by a double filament cell and a cold-lip cell, respectively. A few $\mu \mathrm{m}$ of $\mathrm{GaN}$ is deposited at $820^{\circ} \mathrm{C}$ before growing the QWs structures which are separated by $100 \AA$-thick AlGaN barriers. The $\mathrm{Al}$ mole fraction is measured in situ by the RHEED intensity oscillation technique [11]. The PL experiments are performed at $10 \mathrm{~K}$. Two excitation sources are used, either a HeCd laser $(10 \mathrm{~mW})$ or a frequency-doubled $\mathrm{Ar}^{+}$laser $(244$ $\mathrm{nm} / 40 \mathrm{~mW})$.

\section{RESULTS AND DISCUSSIONS}

The quality of the quantum heterostructures is strongly dependent on the surface morphology. Fig. 1 shows the surface observed by scanning electron microscopy after the growth of a 10 period $\mathrm{Al}_{0.11} \mathrm{Ga}_{0.89} \mathrm{~N} / \mathrm{GaN}$ multi(M)-QW structure. Despite the standard grain-like structure of the $\mathrm{GaN}$ film, the roughness is rather low. Indeed, the rms roughness measured on a $3 \times 3 \mu \mathrm{m}^{2}$ scan area by atomic force microscopy is only $3 \mathrm{~nm}$. This value falls down to $0.2 \mathrm{~nm}$ for $100 \times 100 \mathrm{~nm}^{2}$ scan which is consistent with the observation of RHEED intensity oscillations attesting a 2D layer by layer growth mode. This point constitutes a key issue for achieving AlGaN/GaN QWs with sharp interfaces, but also for measuring in situ the Al mole fraction in the AlGaN barriers.

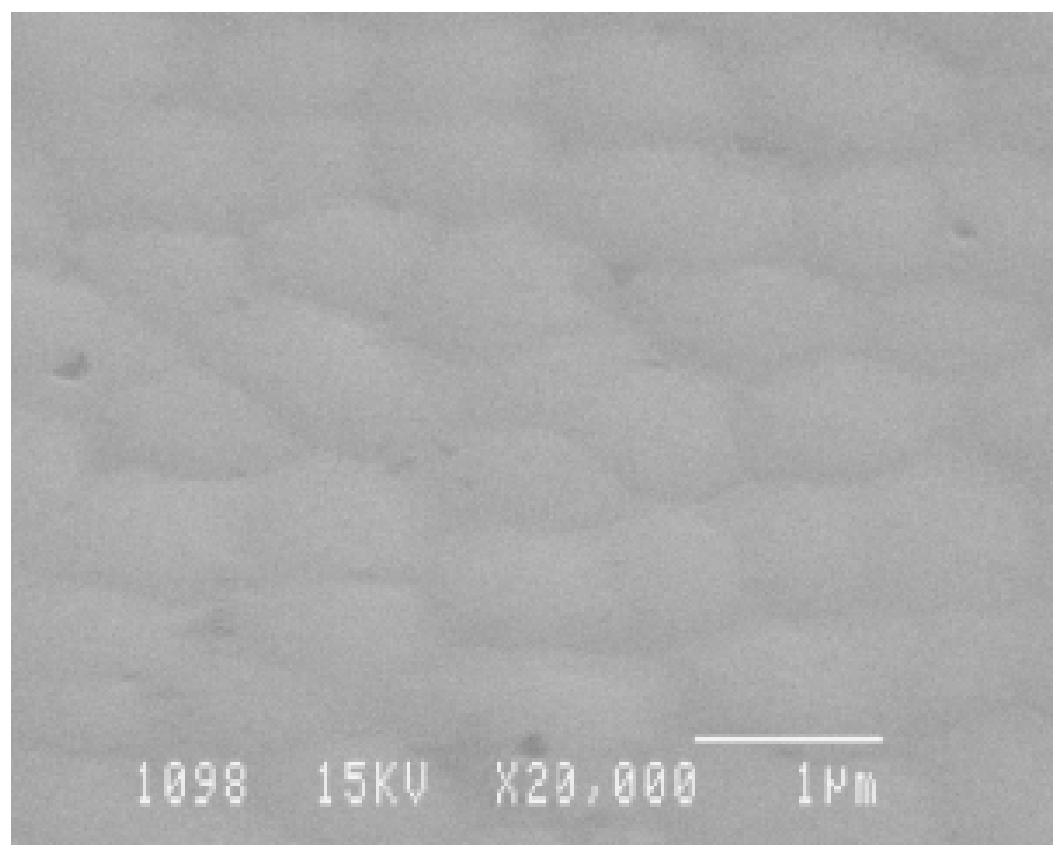

Figure 1: scanning electron micrograph of a multi-quantum well sample grown on sapphire. Fig. 2 displays the intensity variation of the specular beam intensity recorded during the growth of $\mathrm{Al}_{0.1} \mathrm{Ga}_{0.9} \mathrm{~N}$ at $600{ }^{\circ} \mathrm{C}$. The period corresponds to the growth of $1 \mathrm{ML}$ and 
allows one directly determining the growth rate. From the difference with the growth rate of pure $\mathrm{GaN}$, we can measure the $\mathrm{Al}$ mole fraction $\left(\mathrm{x}=\left(\mathrm{r}_{\mathrm{AlGaN}}-\mathrm{r}_{\mathrm{GaN}}\right) / \mathrm{r}_{\mathrm{AlGaN}}\right)$. It should be noted that in order to avoid the flux transient which takes place just after opening the Ga and Al shutters, and which may affect the measurements, a careful procedure has been followed: taking advantage of the stability of $\mathrm{GaN}$ at $600^{\circ} \mathrm{C}$ without ammonia, a general shutter is intercalated between the cells and the sample, and is only opened when the Ga and Al fluxes are stabilized. The data in Fig. 2 correspond to such a procedure. Thick AlGaN layers $(1 \mu \mathrm{m})$ have been grown and energy dispersive $\mathrm{x}$-ray (EDX) has been used to determine the Al mole fraction. The discrepancy between RHEED and EDX determinations is within one percent.

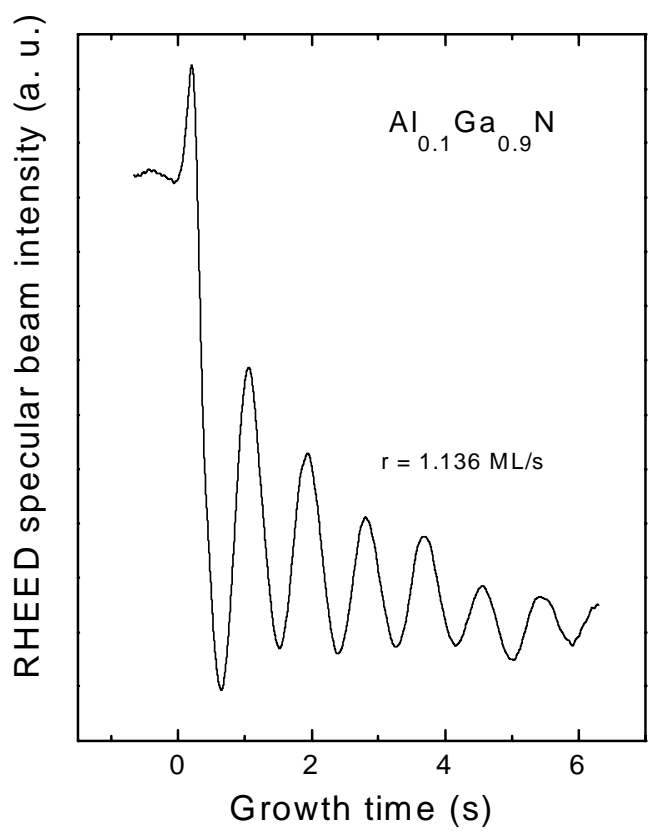

Figure 2: RHEED intensity oscillations recorded during $\mathrm{AlGaN}$ growth.

The $10 \mathrm{~K}$ PL spectra of AlGaN layers with different Al mole fractions are shown in Fig. 3 . The peak linewidth increases with the Al composition together with the Stokes-shift between PL and reflectivity. This indicates that a degradation of the crystal quality occurs. Actually, AlGaN barriers have been grown keeping the same growth parameters than GaN (growth temperature $820^{\circ} \mathrm{C}$ ), while it is obvious that high $\mathrm{Al}$ content AlGaN layers should be grown at higher temperature.

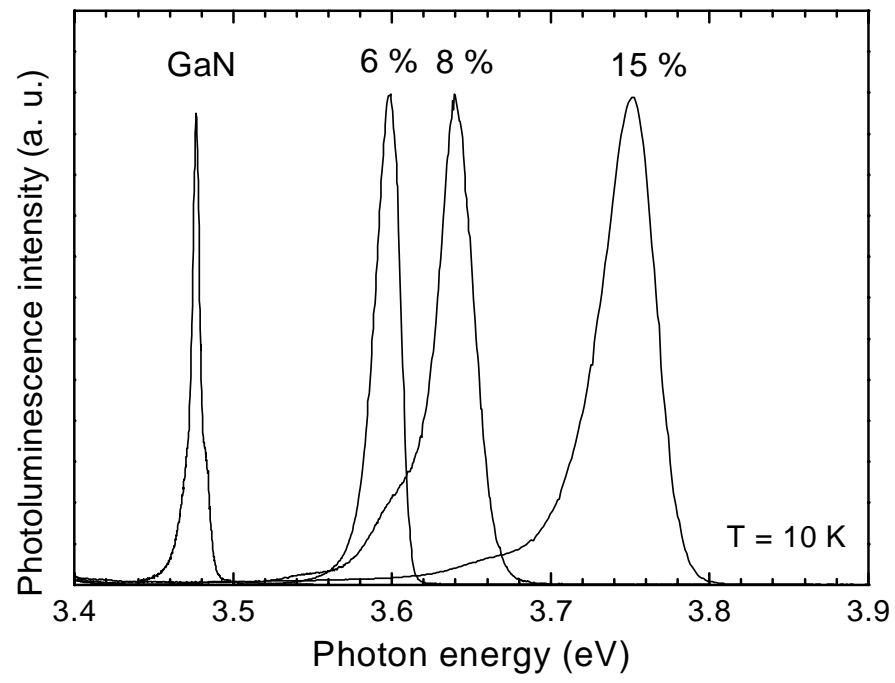

Figure 3: PL spectra of AlGaN layers with different Al mole fraction. 
The structural properties of the quantum heterostructures have been checked by XRD reciprocal space mapping, as shown for instance in Fig. 4 for a MQW sample. This sample consists of 10 periods of $\mathrm{GaN}(6 \mathrm{MLs}) / \mathrm{AlGaN}(19 \mathrm{MLs})$ deposited on a $2 \mu \mathrm{m}$-thick GaN template. Fig. 4 shows that $\mathrm{Al}_{0.11} \mathrm{Ga}_{0.89} \mathrm{~N}$ barriers are pseudomorphically strained onto the $\mathrm{GaN}$ template. Both the value of a and c lattice constants $\left(3.189_{2} \AA\right.$ and $5.185_{5} \AA$, respectively) and the position of the A free exciton $(3.478 \mathrm{eV})$ indicate that the $\mathrm{GaN}$ thick buffer layer is nearly relaxed. AlGaN barriers are thus under tensile stress. This point has to be kept in mind for the following part which concerns the origin of the built-in electric field in AlGaN/GaN Qws.

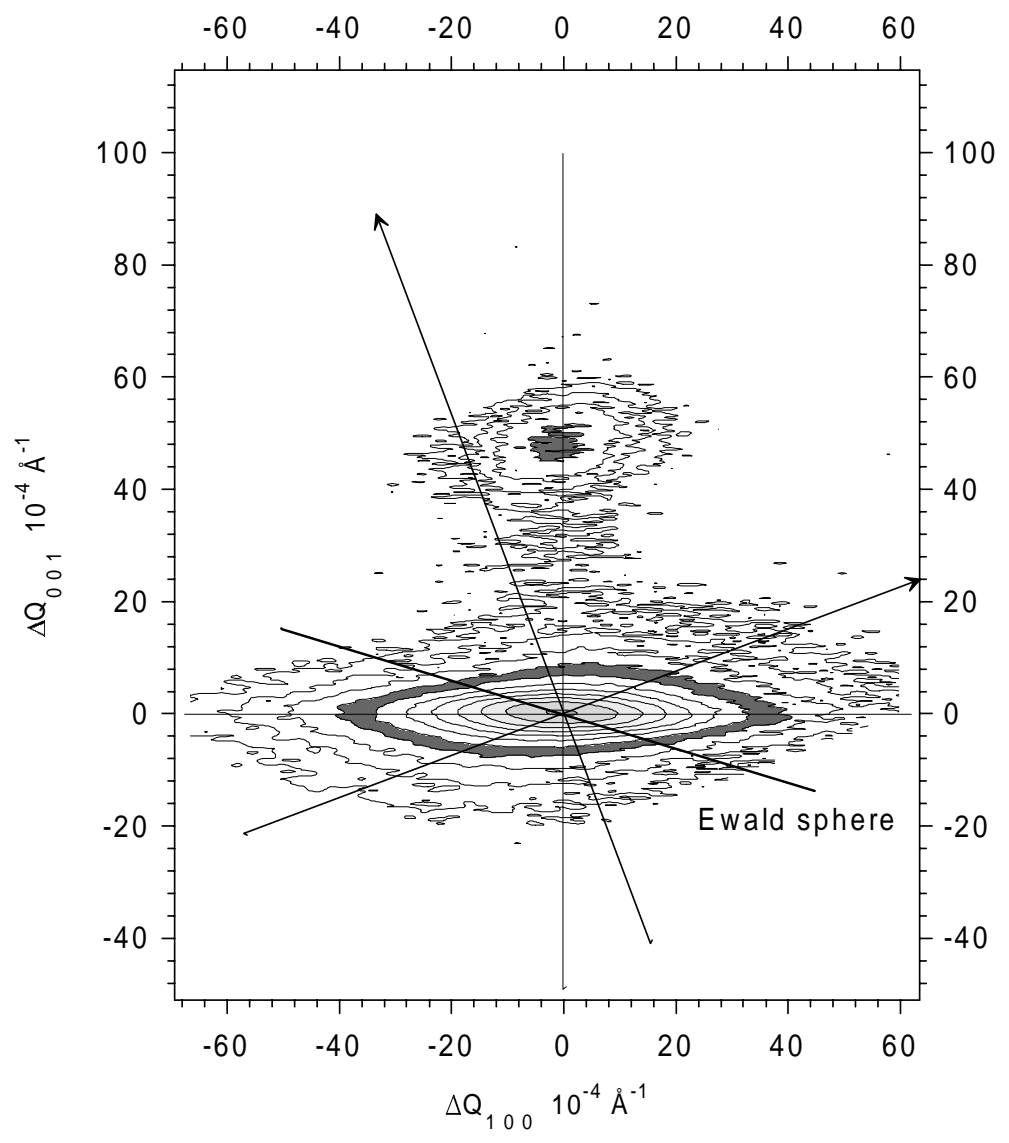

Figure 4: XRD reciprocal space mapping of a $10 \mathrm{xGaN}(6 \mathrm{MLs}) / \mathrm{Al}_{0.11} \mathrm{Ga}_{0.89} \mathrm{~N}(19 \mathrm{MLs}) \mathrm{MQW}$

Let us turn now towards the optical properties of $\mathrm{AlGaN} / \mathrm{GaN}$ based quantum structures. Fig. 5 displays the $10 \mathrm{~K} \mathrm{PL}$ spectra of two samples containing four QWs of different thicknesses with Al composition in the AlGaN barriers of $13 \%$ (a) and $27 \%$ (b), respectively. The thickness of the barriers is $100 \AA$. For the $\mathrm{Al}$ composition of $13 \%$ (Fig. 5(a)), the PL energy of the 17 ML wide well is much lower than the GaN band gap. This is a consequence of a quantum confined Stark effect due to a strong internal electric field in the quantum structure. This effect is reinforced in Fig. 5(b) for an $\mathrm{Al}$ mole fraction in the barriers of 27\%. The $26 \mathrm{meV}$ splitting of the PL line of the 16 MLs wide QW in Fig. 5(b) could correspond to a well thickness fluctuation of $1 \mathrm{ML}$ taking into account a built-in electric field of $1 \mathrm{MV} / \mathrm{cm}$. The values of the internal electric field have been deduced from fitting PL energies with a self-consistent envelope function model including the electric field [7]. They are plotted in Fig. 6 as a function of the Al composition in the barriers from 8 to $27 \%$. The field is shown to vary linearly with Al content, extrapolating to zero for pure $\mathrm{GaN}$. It is larger than $1 \mathrm{MV} / \mathrm{cm}$ for an $\mathrm{Al}$ content of $27 \%$. 
Let us discuss the strength and origin of the internal field. First, we mention that for an Al content in the barriers in the 10-15\% range, Fig. 6 shows that the field is in the $400-600 \mathrm{kV} / \mathrm{cm}$ range. This is in agreement with the results of other authors, who also studied AlGaN/GaN heterostructures with similar barrier compositions [5-8,12]. Second, as exemplified in Fig. 4, we are dealing with strained $\mathrm{AlGaN}$ barriers on nearly relaxed $\mathrm{GaN}$. This means that the piezoelectric effect occurs in our samples mainly in the barrier, and not in the well (as in the typical case of (111) grown GaAs/GaInAs QWs for instance). Moreover, following Bernardini et al. $[9,10]$, we ascribe the Stark effect in our samples to the difference in total (piezoelectric and spontaneous) polarization between well and barrier materials. In that case, the resulting electric field in the well (for infinite barriers, which is not the case of our samples) is given by $\mathrm{F}=\left(\mathrm{P}_{\mathrm{b}^{-}}\right.$ $\left.\mathrm{P}_{\mathrm{w}}\right) / \varepsilon \varepsilon_{0}$, where $\mathrm{P}_{\mathrm{b}}$ and $\mathrm{P}_{\mathrm{w}}$ are the total polarization in the barrier and the well, respectively. Note that with the above definition of the electric field, the effect of a biaxial compressive strain in $\mathrm{GaN}$ is the same as that of a biaxial tensile strain in AlGaN barriers (apart from a small difference due to the slight differences of material constants and that of deformation potentials). When using the theoretical piezoelectric constants and spontaneous polarization values [10], field magnitudes at least a factor of 2 larger than those deduced from figure 6 are obtained. The values are still too large when taking into account the effect of finite barrier width [7]. Apart from the uncertainties in the physical parameters used, reasons for the discrepancies could be the crystal quality (the high dislocation density in $\mathrm{GaN} / \mathrm{Al}_{2} \mathrm{O}_{3}$ samples may influence the strain field), but also partial screening of the polarization field due to the residual n-type doping level of $\mathrm{GaN}$ (and $\mathrm{AlGaN}$ ), in the $10^{17} \mathrm{~cm}^{-3}$ range.

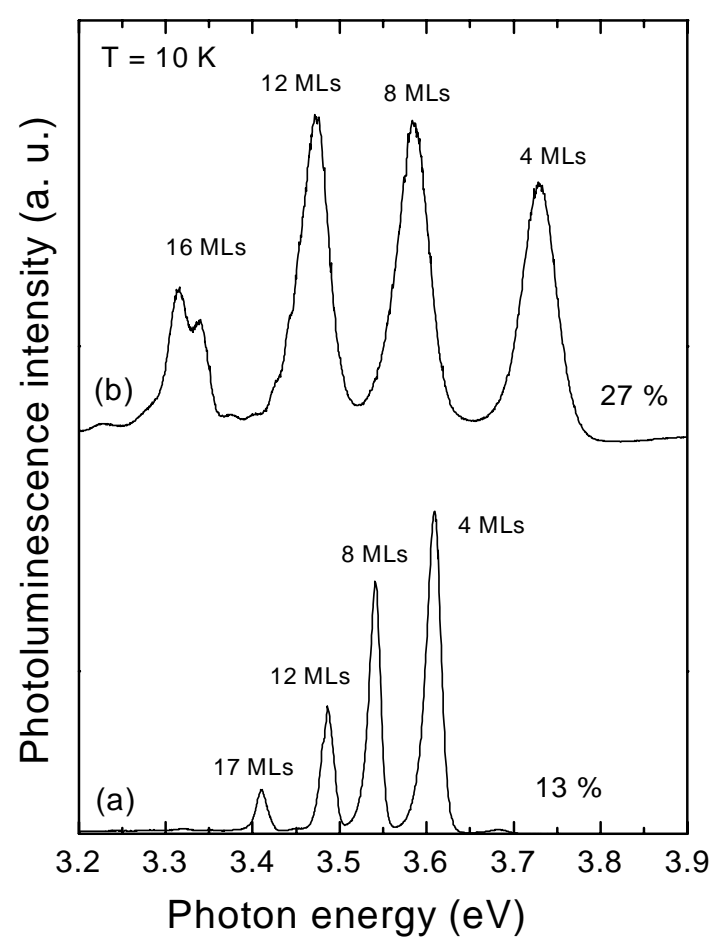

Figure 5: PL spectra of QW structures content

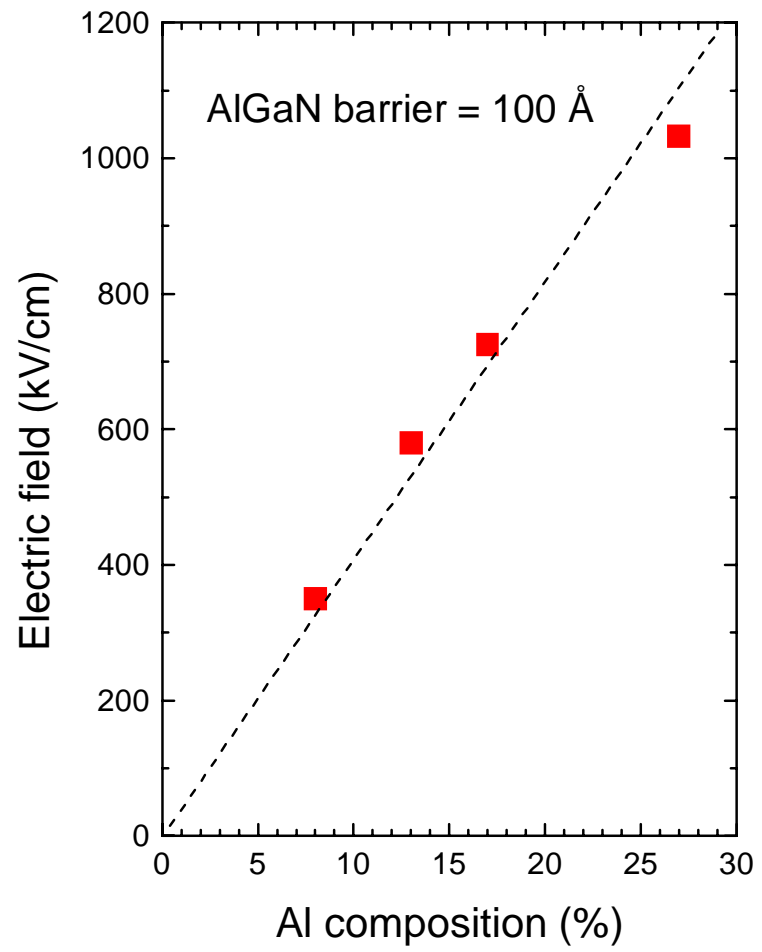

Figure 6: electric field versus the $\mathrm{Al}$

\section{CONCLUSIONS}


$\mathrm{AlGaN} / \mathrm{GaN}$ quantum wells of various widths have been grown by molecular beam epitaxy on cplane sapphire substrates with different Al contents in the barriers. The QW transition energies deduced from low temperature photoluminescence exhibit a quantum confined Stark effect due to strong internal electric fields which can reach $1 \mathrm{MV} / \mathrm{cm}$ for only $25 \%$ of $\mathrm{Al}$ content. This electric field is found to vary linearly with the $\mathrm{Al}$ mole fraction, at least up to $\mathrm{x} \approx 0.3$. A conclusion of this study is that, due to the balance between the blue shift due to quantum confinement (increasing with $\mathrm{Al}$ content in the barrier), and red shift due to the Stark effect (also increasing with $\mathrm{Al}$ content), only $\mathrm{GaN}$ well thicknesses lower than $\approx 11 \mathrm{MLs}(\approx 30 \AA)$ yield transition energies larger than the GaN band gap, at least in the range of barrier thicknesses and AlGaN compositions studied in this work.

\section{ACKNOWLEDGMENTS}

This work was supported in part by E.C. Contract No. BPR-CT96-0334 'ANISET'

\section{REFERENCES}

[1] S. Nakamura and G. Fasol, 'The Blue Laser Diode', Springer - Berlin (1997)

[2] R. Niebuhr, K.H. Bachem, D. Behr, C. Hoffman, U. Kaufmann, Y. Lu, B. Santic, J. Wagner, M. Arlery, J.L. Rouvière and H. Jürgensen, Mat. Res. Soc. Symp. Proc. 449, 769 (1997).

[3] M. Smith, J.Y. Lin, H.X. Jiang, A. Khan, Q. Chen, A. Salvador, A. Botchkarev and H. Morkoç, Mat. Res. Soc. Symp. Proc. 449, 829 (1997).

[4] N. Grandjean and J. Massies, Appl. Phys. Lett., 73, 1260 (1998).

[5] J.M. Im, H. Kollmer, J. Off, A. Sohmer, F. Scholz and A. Angleiter, Mat. Res. Soc. Symp. Proc. 482, 513 (1998)

[6] J.S. Im, H. Kollmer, J. Off, A. Sohmer, F. Scholz, and A. Hangleiter, Phys. Rev. B 57, R9435 (1998)

[7] M. Leroux, N. Grandjean, M. Laügt, J. Massies, B. Gil, P. Lefebvre, and P. Bigenwald, Phys. Rev. B 58, R13371 (1998)

[8] P.Lefebvre, J.Allègre, B.Gil, H.Mathieu, P.Bigenwald, N.Grandjean, M.Leroux and J.Massies, Phys. Rev. B, to be published.

[9] F. Bernardini, V. Fiorentini and D. Vanderbilt, Phys. Rev. B 56, R10024 (1997)

[10] F. Bernardini and V. Fiorentini, Phys. Rev. B 57, R9427 (1998)

[11] N.Grandjean and J.Massies, Appl. Phys. Lett. 71, 1816 (1997)

[12] T.Honda, T.Miyamoto, T.Sakaguchi, H.Kawanishi, F.Koyama and K.Iga, J. Cryst. Growth 189/190, 644 (1998) 